\title{
Deep learning versus traditional methods for parking lots occupancy classification
}

\author{
Mohamed S. Farag, M. M. Mohie El Din, H. A. El Shenbary \\ Department of Mathematics, Faculty of Science Al-Azhar University Cairo, Egypt
}

\begin{tabular}{|c|c|}
\hline Article Info & ABSTRACT \\
\hline Article history: & \multirow{12}{*}{$\begin{array}{l}\text { Due to the increase in number of cars and slow city developments, there is a need for } \\
\text { smart parking system. One of the main issues in smart parking systems is parking } \\
\text { lot occupancy status classification, so this paper introduce two methods for parking } \\
\text { lot classification. The first method uses the mean, after converting the colored image } \\
\text { to grayscale, then to black/white. If the mean is greater than a given threshold it is } \\
\text { classified as occupied, otherwise it is empty. This method gave } 90 \% \text { correct classif- } \\
\text { cation rate on cnrall database. It overcome the alexnet deep learning method trained } \\
\text { and tested on the same database (the mean method has no training time). The second } \\
\text { method, which depends on deep learning is a deep learning neural network consists of } \\
11 \text { layers, trained and tested on the same database. It gave } 93 \% \text { correct classification } \\
\text { rate, when trained on cnrall and tested on the same database. As shown, this method } \\
\text { overcome the alexnet deep learning and the mean methods on the same database. } \\
\text { On the Pklot database the alexnet and our deep learning network have a close resutls, } \\
\text { overcome the mean method (greater than } 95 \% \text { ). }\end{array}$} \\
\hline Received Jan 5, 2020 & \\
\hline Revised Mar 62020 & \\
\hline Accepted Mar 20, 2020 & \\
\hline & \\
\hline Keywords: & \\
\hline Alexnet & \\
\hline Deep learning & \\
\hline DWT & \\
\hline Lots classification & \\
\hline PCA & \\
\hline Smart Parking & \\
\hline
\end{tabular}

\section{Corresponding Author:}

Mohamed S. Farag,

Department of Mathematics, Faculty of Science,

Al-Azhar University,

Nasr city, 11884, Cairo, Egypt. Tel: 0020-1006-574-243.

E-mail: mohamed.s.farag@azhar.edu.eg

\section{INTRODUCTION}

The industrialization of the world, slow paced city development, and increase in number of cars has resulted parking problems. There is need for an intelligent system to be used for allocating free parking lots. Smart Parking System(SPS), is shown as a small version of an Intelligent Transportation Systems (ITS) [1]. Using Internet of Things (IoT), to minimize the traffic and parking congestion. One of the most challenge problem is the way to detect a parking lot state( Occupied, or Free). Smart parking systems based on emergency status was proposed using FPGA to perform a lot of tasks like automatic parking depends on the behavior of driving and warning the drivers [2]. An automated parking management system,(APMS) for recognizing vehicles plate numbers in [3] based on template matching . In [4] the proposed work focused on providing a solution to vehicle parking Management System. The proposed work is developed using Ultrasonic Sensors, Arduino Mega, Android, Wi-Fi Module and Google maps. The proposed system is designed to detect the vacant parking slots through the IOT technology utilizing Google maps and Android application. The Wi-Fi Module is used to send the information to the server. Authors in [5] presented a multi-camera system for the management of vacant parking places by means of vehicle detection and mapping it into the parking spaces of a parking lot. The system achieved $90 \%$ correct classification rate. Authors in [6-10] viewed a lot of smart parking systems. A crowd of taxis to sense onstreet parking space availability was developed in [11]. A supervised learning method was developed in 
[12] to estimate parking occupancy status in road side using mobile sensing approach. Multiple road tests were conducted around Oxfordshire and Guildford in the U.K. The advantage of the mobile sensing approach is that it requires a significantly smaller number of sensor units compared with the fixed sensing solutions. To cover 8000 parking spaces, 132 mobile sensing units compared with 12000 fixed sensors. In the case of exact GPS readings, followed by a map matching technique, the classification rate of the system was above $90 \%$. The mobile sensing system becomes more pronounced as the parking lots number to be monitored increases. A mobile AR-based interactive smart parking system was applied in [13]. The parking system in a city which is embedded with various features like automated, rotary parking and nearest parking slot allotment using IoT and sensor technology was discussed by authors in [14]. A literature over the period of 2000-2016 on parking solutions as they were applied to smart parking development and evolution, and propose three macro-themes: information collection, system deployment, and service dissemination was reported in [15]. In [16, 17] authors developed a smart parking system based on fog computing, enabled a fog for efficient car parking architecture. The video analysis method has the advantages of easy installation, saving hardware cost and extending other functions compared with the sensing coil detection and the infrared detection. The boundary coordinates and central coordinates of the license plate region are used to classify the occupancy status of the parking space. Authors in [18] reported that the classification rate of the parking space detection system is beyond $90 \%$.

The remainder of this article is shown as follows. Section 2 view the standard methods used for lot classification. Sections 3, 4 presents the databases used for training and testing and traditional methods results. Section 5 view the proposed method and its results compared with the alexnet and the mean method results. The Conclusion and our future work are shown in Section 6.

\section{STANDARD METHODS}

\subsection{Principal component analysis}

The principal component analysis, (PCA) is considered to be a statistical model used for feature extraction, one of the most used and successful techniques in image processing. PCA is mainly used for reducing dimensionality of the row data space to the smaller dimensionality of the feature space. This reduction is confirmed by the linear transformation

$$
Z=A Y
$$

Where Z, A, Y are the feature matrix,transformation matrix and original image respectively. PCA can give us data prediction, compression, redundancy removal, and feature extraction. The scope of using PCA for lots occupancy detection is to express the large one dimensional vector of pixel constructed from the two dimensional lot image into the feature space(Principal Components). It is known as eigenspace projection. Eigenspace can be computed by calculating the eigenvectors of the corresponding covariance matrix of the training images. In 1991, PCA method was firstly proposed by M. Turk and A. Pentland [19].

Assume we have a dataset of $\mathrm{N}$ slot images $Y_{1}, Y_{2}, \ldots, Y_{N}$. Originally, each image is a 2-dimentional matrix of size $\mathrm{n}$ by $\mathrm{m}$. By converting each 2-dimenstional image to 1-dimentional column vector of size $n \times m$ as follows.

$$
Y_{i}=\left(\begin{array}{c}
y_{1} \\
y_{2} \\
\cdot \\
\cdot \\
\cdot \\
y_{n m}
\end{array}\right)
$$

The images set will be

$$
Y=\left[Y_{1}, Y_{2}, \ldots Y_{N}\right]
$$

then, the mean image $\mathrm{Y}_{m}$ is computed as follows

$$
Y_{m}=\frac{1}{N} \sum_{i=1}^{N} Y_{i}
$$


and the covariance matrix of the dataset is given by the formula

$$
C=\frac{1}{N} \sum_{i=1}^{N}\left(Y_{i}-Y_{m}\right)\left(Y_{i}-Y_{m}\right)^{T} \text {. }
$$

Let $M_{i}=\left(Y_{i}-Y_{m}\right)$, be the centered image. Now we want to compute eigenvectors $e_{i}$ and the eigenvalues $\lambda_{i}$ of this covariance matrix.

$$
C=M M^{T}
$$

Now, the size of $\mathrm{C}$ is $n m \times n m$, so image of size $100 \times 100$ will give a covariance matrix of size $10000 \times 10000$ which will not be practical to solve for the eigenvectors of $\mathrm{C}$ directly. Let $\mu_{i}, d_{i}$ be the eigenvectors and eigenvalues of $M^{T} M$, respectively.

That is mean that

$$
M^{T} M d_{i}=\mu_{i} d_{i}
$$

By multiplying both sides by $\mathrm{M}($ from left)

$$
\left(M M^{T}\right) M d_{i}=\mu_{i}\left(M d_{i}\right)
$$

The first $N-1$ eigenvalues $\lambda_{i}$ and eigenvectors $e_{i}$ of the covariance matrix $C=M M^{T}$ are given by $M d_{i}$ and $\mu_{i}$, respectively. $M d_{i}$ needs to be normalized in order to be equal to $e_{i}$. The transformation matrix A can be constructed from the $\mathrm{k}$ eigenvectors corresponding to the $\mathrm{k}$ largest eigenvalues of the desired covariance matrix.

\subsection{Euclidean distance}

Euclidean distance is used to classify the images in the test image set for which class it belong to. Comparing the weight matrix (feature vectors) of the images in the training set with the corresponding weight matrix of the test image using euclidean distance,

$$
\varepsilon_{i}=\left\|\Omega_{T}-\Omega_{i}\right\|
$$

where $\Omega_{i}$ is a vector describing the $i_{t h}$ image in the training set.

\subsection{Discrete wavelet transform}

Wavelet transforms are considered to be a mathematical functions used to convert row data to frequency components, each component is treated with a resolution according to its scale. Wavelets were presented in the field of electrical engineering, mathematics and quantum physics [20]. In the last decades, many new wavelet applications were introduced like Earthquake predictions, image compression, human vision and radar. For an image, the wavelet decomposition function is defined as follows:

$$
\Psi_{V, U(t)}=\sum_{x=0}^{N-1} \sum_{y=0}^{N-1} g(x, y) \exp ^{-j \pi \frac{(V x+U y)}{N}}
$$

where, the Kernel function is:

$$
\begin{gathered}
\exp ^{-j \pi \frac{(V x+U y)}{N}}, \\
g(x, y) \text { is a 2D image, }
\end{gathered}
$$

and $N$ is the number of pixels in the desired image.

The Wavelet transform is considered to be a useful computational tool for signal and image processing applications. DWT is used in a wide range in pattern recognition area [21-23]. DWT generates 4 coefficients in each level decomposition. Approximation, Horizontal, vertical and Diagonal information. The approximation coefficient of the 1st level decomposition is treated as the original image, because it contains more information about the image. 


\subsection{RGB to gray conversion}

To convert a colored images to grayscale, we use the following equation.

$$
X=0.2989 * R+0.5870 * G+0.1140 * B .
$$

According to a threshold value $\theta$, the gray image pixels value is converted to a black and white( 0 or 1$)$ according to the shown equation

$$
Y_{i j}= \begin{cases}1 & \text { where } X_{i j}>=\theta \\ 0 & \text { otherwise }\end{cases}
$$

\section{DATABASE DESCRIPTION}

\subsection{PKLOT database}

The PKLot database contains 12,417 images of parking images and 695,899 images of parking spaces segmented from them, checked and labeled manually. Images were captured at the parking lots of the Federal University of Parana (UFPR) and the Pontical Catholic University of Parana (PUCPR), both located in Curitiba, Brazil [24]. Table 1 views number of free and busy spaces in the pklot dataset.

\begin{tabular}{|c|c|c|c|c|c|c|}
\hline Parking lot & $\begin{array}{l}\text { Weather } \\
\text { condition }\end{array}$ & No of days & No of images & No of occupied & No of empty & total \\
\hline \multirow{4}{*}{$\begin{array}{l}\text { UFPR04 } \\
\text { (28 parking spaces ) }\end{array}$} & Sunny & 20 & 2098 & $32166(54.98 \%)$ & $26334(45.02 \%)$ & 58400 \\
\hline & Overcast & 15 & 1408 & $11608(29.47 \%)$ & $27779(70.53 \%)$ & 39387 \\
\hline & Rainy & 14 & 285 & $2351(29.54 \%)$ & $5607(70.46 \%)$ & 7958 \\
\hline & Subtotal & & 3791 & $46125(43.58 \%)$ & $59720(56.42 \%)$ & 105845 \\
\hline UFPR05 & Sunny & 25 & 2500 & $57584(57.65 \%)$ & $42306(42.35 \%)$ & 99890 \\
\hline \multirow[t]{3}{*}{ (45 parking spaces ) } & Overcast & 19 & 1426 & $33764(59,27 \%)$ & $23202(40.73 \%)$ & 56966 \\
\hline & Rainy & 8 & 226 & $6078(68.07 \%)$ & $2851(31.93 \%)$ & 8929 \\
\hline & Subtotal & & 4152 & $97426(58.77 \%)$ & $68359(41.23 \%)$ & 165785 \\
\hline PUCPR & Sunny & 24 & 2315 & $96762(46.42 \%)$ & $\begin{array}{l}111672 \\
(53.58 \%)\end{array}$ & 208433 \\
\hline \multirow[t]{4}{*}{ (100 parking spaces ) } & Overcast & 11 & 1328 & $42363(31.90 \%)$ & $90417(68.10 \%)$ & 132780 \\
\hline & Rainy & 8 & 831 & $55104(66.35 \%)$ & $27951(33.65 \%)$ & 83056 \\
\hline & Subtotal & & 4474 & $\begin{array}{l}194229 \\
(45.78 \%)\end{array}$ & $\begin{array}{l}230040 \\
(51.46 \%)\end{array}$ & 424269 \\
\hline & TOTAL & & 12417 & $\begin{array}{l}337780 \\
(48.54 \%)\end{array}$ & $\begin{array}{l}358119 \\
(51.46 \%)\end{array}$ & 695899 \\
\hline
\end{tabular}

Table 1. PKLot characteristics

\subsection{CNRPark database}

CNRPark is a new dataset consists of 12,000 images captured in different days of November 2015 to February 2016 under different weather conditions by 9 cameras with various angles of view and perspectives. It has different situations of light conditions, shadowed cars, and includes obstacles like (lampposts, trees, and other cars). The segmented patches (images) of parking lots belonging to the CNRPark subset have size $(150 * 150)$ pixel. Images of a real parking slots in different days, with different light and weather conditions, contains images with high variability related to occlusions, which makes this dataset more compatible with a real state of an outdoor parking slot [25]. Table 2 views number of free and busy spaces in the desired dataset.

Table 2. CNRPark dataset

\begin{tabular}{llll}
\hline Dataset & Free Spaces & Busy Spaces & Total \\
\hline CNRPark & 4181 & 8403 & 12,584 \\
CNRPark-EXT & 65,658 & 79,307 & 144,965 \\
\hline
\end{tabular}




\section{TRADITIONAL METHODS RESULT}

Figure 1 and Figure 2 view the steps of training and testing stages using DWT, PCA and Euclidean Distance. Table 3 views the results of the above methods using different training, testing percentage number of images on the PKLot and CNR databases. It is clear from the results that using 1 level DWT before applying PCA increased the correct classification rate. in case of training $80 \%$ of the dataset DWT_ PCA reach a high correct classification rate $80 \%$.

Another method was applied without training stage. This method convert the rgb image to a grayscale using 11, then convert it to a black and white according to a threshold 12 . Then compute the mean of the image. If the mean $; 0.575$ the image is classified as occupied slot, else it is free. This method gave average $90 \%$ correct classification rate. This classification rate outperform the previous mentioned methods(PCA, DWT+PCA) and save time(there is no time for training).

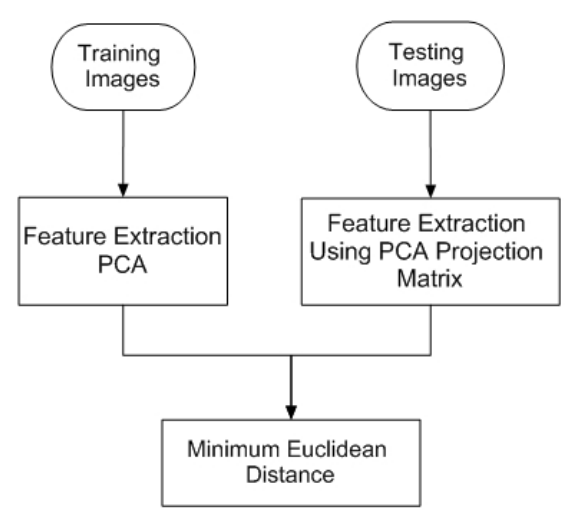

Figure 1. Training and testing using PCA

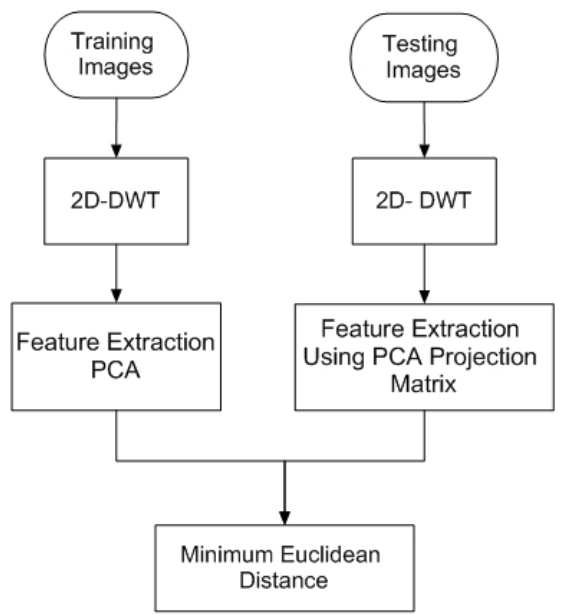

Figure 2. Training, testing using DWT and PCA

Table 3. Classification results using DWT and PCA

\begin{tabular}{ccccc}
\hline No Training images \% & No Testing images \% & PCA & DWT+PCA & RGB2Gray+BW+Mean (our Method) \\
\hline 10 & 90 & 63 & 71 & 89 \\
20 & 80 & 75 & 75 & 89 \\
30 & 70 & 40 & 59 & 89 \\
40 & 60 & 75 & 65 & 89 \\
50 & 50 & 25 & 76 & 89 \\
60 & 40 & 34 & 50 & 89 \\
70 & 30 & 73 & 53 & 89 \\
80 & 20 & 65 & 80 & 89 \\
90 & 10 & 40 & 70 & 90 \\
\hline
\end{tabular}

\section{RESEARCH METHODS}

Deep Learning is a branch of Artificial Intelligence, aims for developing techniques that allow computers to learn complex perception tasks, such as seeing and hearing, at high level of accuracy. It provides near-human level accuracy in object detection, image classification, speech recognition, vehicle detection, language processing, and etc. The traditional approaches to the classification problem use ad-hoc functions to extract from an image specific features that are considered to be indicative of certain objects. The outputs of these feature extraction functions are then given in input to a classification function, which determines whether or not a particular object was detected. However, this approach leads to low and false-alarm prone detectors. In addition, it presents the following problems:

(a) It is hard to think of general, reliable features, robust, which map to specific object types.

(b) It is a huge task to determine the right combination of features for each type of object to detect.

(c) It is difficult to design functions that are robust to rotations, translations and scaling of objects. 
All these problems make developing high object detection accuracy and classifying very hard. The Deep Learning technique, exploits a high number of labeled data to know which features and combinations of it are most describing for each class of objects to be classified, and develop a combined feature classification and extraction model. This model could be developed not only to classify objects trained on it, but also unseen objects similar to them. A Deep Learning method particularly impacted for vision tasks using Constitutional Neural Networks (CNN) [26].

A CNN is consisted of a large number of hidden layers, to perform mathematical computations on the input provided by the previous layer and generate an output, which is given as input to the following layer Figure 3. A CNN differs from neural networks for the presence of convolutional layers, which can be a good model and discern correlation of neighboring pixels rather than fully connected layers. To classify inputs, the final outputs of the CNN will be the label of classes the network has been trained. The training stage is usually extremely costing from a computational point of view, and may take a long time to complete. After the network training stage has been completed and the classifier has been initialized accordingly, the time for prediction stage is quite fast and efficient.

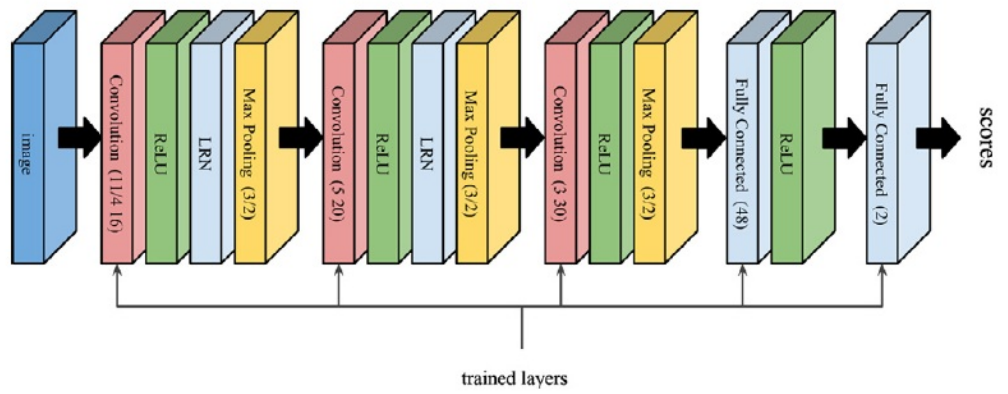

Figure 3. CNN architecture

\subsection{AlexNet vs mean}

AlexNet is considered to be a convolutional neural network, had a high impact on the machine learning field, specially in the application of deep learning to machine vision. It famously won the 2012 ImageNet LSVRC-2012 competition by a large margin (15.3\% VS 26.2\% (second place) error rates) [27]. Figure 4 shows the architecture of the AlexNet. AlexNet contains 8 hidden layers. There exist 5 convolutional layers followed by 3 fully connected layers. Rectified Linear Unit (ReLU) applied after all convolutional and fully connected layers to quick the train. Dropout applied before both the first and the second fully connected year. To train the network, alexnet images were down-sampled to $256 * 256$ pixels and subtraction of the mean activity over the training set from each pixel. 1.2 million training images were used, 50000 images for validation, and 150000 image for testing. The images were classified to 1000 categories, each category have 1000 images.

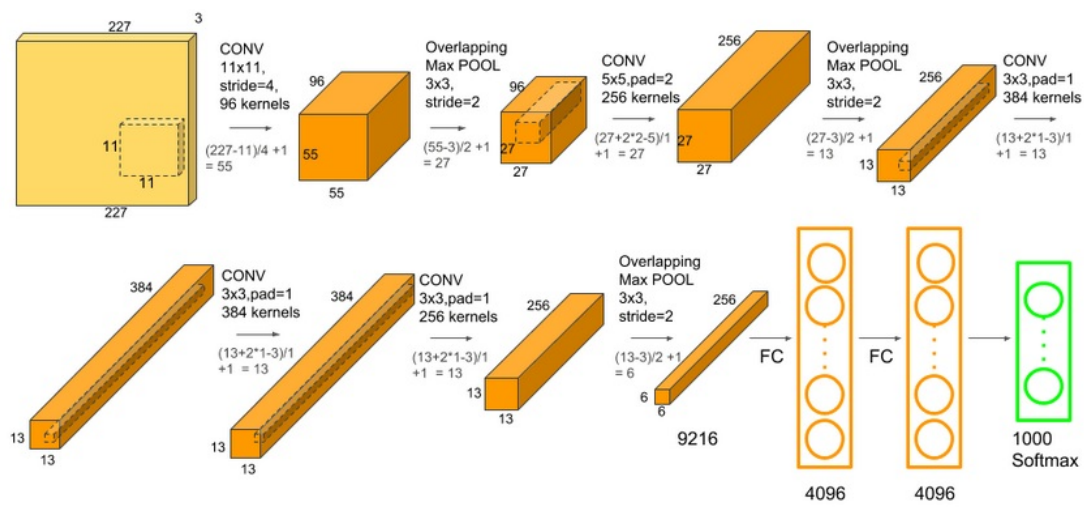

Figure 4. AlexNet architecture 
Table 4 shows the classification results when training AlexNet on 12000 images from the cnrall database. It is shown that the results of the testing pklot sunny gave $83 \%$ correct classification rate.

Table 4. Training 12000 images of cnrall alexnet deep learning

\begin{tabular}{lcc}
\hline Testing Database & Classification rate & Time per image(seconds) \\
\hline Pklot cloudy & $79 \%$ & 0.132 \\
Pklot rainy & $75 \%$ & 0.135 \\
Pklot sunny & $83 \%$ & 0.135 \\
Cnrall & $81 \%$ & 0.133 \\
\hline
\end{tabular}

Table 5 shows the classification results when training AlexNet on 12000 images from the pklot rainy database and testing using the other sets(cnrall, pklot cloudy, sunny, rainy). In the testing stage using pklot cloudy and sunny the AlexNet gave high recognition rate $(98.5 \%$ and $98.8 \%)$.

Table 5. Training 12000 images of pklot rainy alexnet deep learning

\begin{tabular}{lcc}
\hline Testing Database & Classification rate & Time per image(seconds) \\
\hline Pklot cloudy & $98.52 \%$ & 0.133 \\
Pklot rainy & $75 \%$ & 0.135 \\
Pklot sunny & $98.8 \%$ & 0.132 \\
Cnrall & $89 \%$ & 0.136 \\
\hline
\end{tabular}

Table 6 shows the classification results when training AlexNet on 12000 images from the pklot sunny database and testing using the other sets (cnrall, pklot cloudy, sunny, rainy). In the testing stage using pklot cloudy and rainy the AlexNet method gave $(97 \%$ and $94.6 \%)$ correct classification rate.

Table 6. Training 12000 images of pklot sunny alexnet deep learning

\begin{tabular}{lcc}
\hline Testing Database & Classification rate & Time per image(seconds) \\
\hline Pklot cloudy & $97 \%$ & 0.128 \\
Pklot rainy & $94.6 \%$ & 0.130 \\
Pklot sunny & $88 \%$ & 0.129 \\
Cnrall & $87 \%$ & 0.132 \\
\hline
\end{tabular}

Table 7 shows the classification results when training AlexNet on 12000 images from the pklot cloudy database and testing using the other sets (cnrall, pklot cloudy, sunny, rainy). In the testing stage using pklot cloudy and rainy the AlexNet method gave ( $100 \%$ ) correct classification rate.

Table 7. Training 12000 images of pklot cloudy alexnet deep learning

\begin{tabular}{lcc}
\hline Testing Database & Classification rate & Time per image(seconds) \\
\hline Pklot cloudy & $100 \%$ & 0.132 \\
Pklot rainy & $100 \%$ & 0.134 \\
Pklot sunny & $100 \%$ & 0.130 \\
Cnrall & $87 \%$ & 0.14
\end{tabular}

Table 8 shows the results of classifying images using the mean method, DWT 1 level + mean and DWT 2 levels + mean. From the previous results, it is clear that the AlexNet outperform the mean method when testing the pklot database. When classifying the cnrall database, the mean method outperform the Alexnet ( $90 \%$ correct classification rate). Also, the mean method has no training time like the Alexnet method.

Table 8. Classification of Pklot database and cnrall using Mean

\begin{tabular}{lllllll}
\hline $\begin{array}{l}\text { Testing } \\
\text { Database }\end{array}$ & $\begin{array}{l}\text { Rate using } \\
\text { RGB2Gray + } \\
\text { BW+Mean }\end{array}$ & $\begin{array}{l}\text { Time } \\
\text { (seconds) }\end{array}$ & $\begin{array}{l}\text { Rate using DWT 1 } \\
\text { level+RGB2Gray+ } \\
\text { BW+Mean }\end{array}$ & $\begin{array}{l}\text { Time } \\
\text { (seconds) }\end{array}$ & $\begin{array}{l}\text { Rate using DWT 2 } \\
\text { level } \\
\text { +RGB2Gray } \\
\text { +BWean }\end{array}$ & $\begin{array}{l}\text { Time } \\
\text { (seconds) }\end{array}$ \\
\hline Pklot cloudy & $90 \%$ & 0.0042 & $90 \%$ & 0.0106 & $90 \%$ & 0.0106 \\
Pklot rainy & $79 \%$ & 0.0040 & $80 \%$ & 0.0076 & $81 \%$ & 0.0099 \\
Pklot sunny & $83 \%$ & $0.0042 \%$ & $85 \%$ & 0.0092 & $85 \%$ & 0.0104 \\
Cnrall & $90 \%$ & 0.0049 & $86 \%$ & 0.0135 & $86 \%$ & 0.0154 \\
\hline
\end{tabular}




\subsection{Proposed deep learning neural network}

Due to the previous low classification rate showed using AlexNet and the mean method, we propose a deep learning neural network, consists of 11 layers as shown in figure 5. Layer 1 is considered as the input image layer, the input image size is $(150 * 150 * 3)$. layer 2 is a $2 \mathrm{~d}$ convolutional layer, $11 \times 11$ convolutions with stride [1 1], followed by a $3 \times 3$ max pooling layer with stride [2 2]. layer 4 is a 2 d convolutional layer, $5 \times 5$ convolutions with stride [1 1], followed by a $3 \times 3$ max pooling layer with stride [2 2]. layer 6 is a $2 d$ convolutional layer, $5 \times 5$ convolutions with stride [1 1], followed by a Rectified Linear Unit (RelU) layer in order to quick the train. Then dropout layer is used followed by the fully connected layer. layer 10 is the softmax layer followed by the classification output layer. In the beginning 1 convolution layer was used, learning rate was 0.001 , this make the training time is very high. But when we used 3 convolution layers and learning rate 0.00001 the training time was decreased.

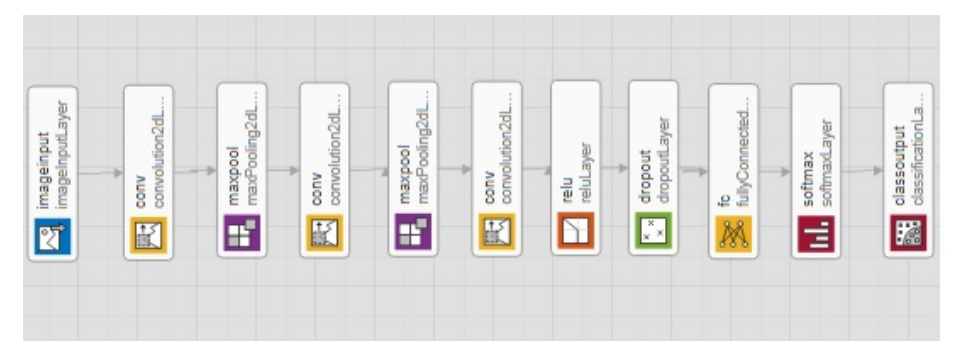

Figure 5. proposed Deep Learning Neural Network Design

Table 9 shows the classification results when training the proposed network on 12000 images from the pklot sunny database and testing using the other sets (cnrall, pklot cloudy, sunny, rainy). In the testing stage using pklot sunny, rainy, and cloudy the proposed network gave over ( $97 \%$ ) correct classification rate, but the classification rate is low in case of cnrall database. Table 10 shows the classification results when training the proposed network on 12000 images from the pklot cloudy database and testing using the other sets (cnrall, pklot cloudy, sunny, rainy). In the testing stage using pklot sunny, rainy, and cloudy the proposed network gave over ( $94 \%$ ) correct classification rate, and the classification rate was increased in case of cnrall database $84 \%$.

Table 9. Training 12000 images of pklot sunny proposed deep learning network

\begin{tabular}{lc}
\hline Testing Database & Classification rate \\
\hline Pklot cloudy & $98 \%$ \\
Pklot rainy & $97 \%$ \\
Pklot sunny & $99 \%$ \\
Cnrall & $79 \%$ \\
\hline
\end{tabular}

Table 10. Training 12000 images of pklot cloudy proposed deep learning network

\begin{tabular}{lc}
\hline Testing Database & Classification rate \\
\hline Pklot cloudy & $99 \%$ \\
Pklot rainy & $95 \%$ \\
Pklot sunny & $94 \%$ \\
Cnrall & $84 \%$ \\
\hline
\end{tabular}

Table 11 shows the classification results when training the proposed network on 12000 images from the pklot rainy database and testing using the other sets (cnrall, pklot cloudy, sunny, rainy). In the testing stage using pklot rainy, and cloudy the proposed network gave over ( $95 \%$ ) correct classification rate, but the classification rate is low in case of cnrall, sunny database. Table 12 shows the classification results when training the proposed network on 12000 images from the cnrall database and testing using the other sets (cnrall, pklot cloudy, sunny, rainy). In the testing stage using pklot rainy, cloudy and rainy the proposed network gave a range ( $80: 85 \%$ ) correct classification rate, but the classification rate increased to $93 \%$ using the cnrall database. Compared with alexnet (which gave 81:89\%) and the mean method (which gave average 90\%), the proposed Deep Learning outperform the desired methods in case of training on the cnrall database (gave 93\%). Also, when training on the pklot database (rainy, cloudy, sunny), it gave classification rate (94: $99 \%$ ), which is acceptable compared with alexnet deep learning method. One of the main reasons for this low rate is the size of images in the pklot database. In pklot database image size is (average 40*40) and the cnrall is (average 150*150), before testing the pklot all images must be resized to $150 * 150$ to be with the same size of the input layer of the proposed deep learning network. 
Table 11. Training 12000 images of pklot rainy

\begin{tabular}{lc}
\multicolumn{2}{c}{ proposed deep learning network } \\
\hline Testing Database & Classification rate \\
\hline Pklot cloudy & $95 \%$ \\
Pklot rainy & $99 \%$ \\
Pklot sunny & $89 \%$ \\
Cnrall & $80 \%$
\end{tabular}

Table 12. Training 12000 images of Cnrall database

\begin{tabular}{lc}
\multicolumn{2}{c}{ proposed deep learning network } \\
\hline Testing Database & Classification rate \\
\hline Pklot cloudy & $85 \%$ \\
Pklot rainy & $82 \%$ \\
Pklot sunny & $80 \%$ \\
Cnrall & $93 \%$ \\
\hline
\end{tabular}

\section{CONCLUSION}

In this paper, we present two methods for parking lot occupancy detection. The first method as shown convert the colored lot image to grayscale, then to black/white and compute the mean of the resulted image. Classifying the image to occupied or empty according to a threshold. This method reported a $90 \%$ correct rate on cnrall database, which overcome the showed methods (alexnet, traditional methods) and has no training time. The second method depends on deep learning techniques. As viewed it is a deep learning network consisting of 11 layers, 3 of them are convolution layers with different kernel size. This method gave 93\% correct classification rate on cnrall database, which overcome the alexnet trained on the same database and the mean method. Training and testing on pklot database, the deep learning methods (alexnet and the proposed deep learnng method) have a closest classification rate and overcome the mean method.

\section{REFERENCES}

[1] Faheem, S. A. Mahmud, G. M. Khan, M. Rahman, and H. Zafar, "A survey of intelligent car parking system," Journal of Applied Research and Technology, vol. vol.11, pp. 714 - 726, 2013.

[2] K. J. Yong and M. H. Salih, "Design and implementation of embedded auto car parking system using fpga for emergency conditions," Indonesian Journal of Electrical Engineering and Computer Science (IJEECS), vol. Vol. 13, No. 3, pp. 678 - 883, 2019.

[3] A. Singh and S. P. Vaidya, "Automated parking management system for identifying vehicle number plate," Indonesian Journal of Electrical Engineering and Computer Science (IJEECS), vol. Vol. 13, No. 1, pp. $77-84,2019$.

[4] Y. S. A. Waili, S. M. Hussain, K. M. Yusof, S. A. Hussain, R. Asuncion, and A. Frank, "Iot based parking system using android and google maps," International Journal of Applied Engineering Research, vol. vol. 13, No. 20, pp. 14689-14697, 2018.

[5] R. Martín Nieto, . García-Martín, A. G. Hauptmann, and J. M. Martínez, "Automatic vacant parking places management system using multicamera vehicle detection," IEEE Transactions on Intelligent Transportation Systems, vol. vol. 20, No. 3, pp. 1069-1080, 2019.

[6] A. Somani, S. Periwal, K. Patel, and P. Gaikwad, "Cross platform smart reservation based parking system," 2018 International Conference on Smart City and Emerging Technology (ICSCET), vol. pp. 1-5, 2018.

[7] T. Kiliç and T. Tuncer, "Smart city application: Android based smart parking system," 2017 International Artificial Intelligence and Data Processing Symposium (IDAP), vol. pp. 1-4, 2017.

[8] S. Kazi, S. Khan, U. Ansari, and D. Mane, "Smart parking based system for smarter cities," 2018 International Conference on Smart City and Emerging Technology (ICSCET), vol. PP. 1-5, 2018.

[9] T. O. Olasupo, C. E. Otero, L. D. Otero, K. O. Olasupo, and I. Kostanic, "Path loss models for low-power, low-data rate sensor nodes for smart car parking systems," IEEE Transactions on Intelligent Transportation Systems, vol. Vol. 19, No. 6, PP. 1774-1783, 2018.

[10] J. Ni, K. Zhang, Y. Yu, X. Lin, and X. Shen, "Privacy-preserving smart parking navigation supporting efficient driving guidance retrieval," IEEE Transactions on Vehicular Technology, vol. Vol. 67, No. 7, PP. 6504-6517, 2018.

[11] F. Bock, S. Di Martino, and A. Origlia, "Smart parking: Using a crowd of taxis to sense on-street parking space availability," IEEE Transactions on Intelligent Transportation Systems, vol. Vol. 21, No. 2, PP. 496-508, 2020.

[12] C. Roman, R. Liao, P. Ball, S. Ou, and M. de Heaver, "Detecting on-street parking spaces in smart cities: Performance evaluation of fixed and mobile sensing systems," IEEE Transactions on Intelligent 
Transportation Systems, vol. vol. 19, No. 7, pp. 2234-2245, 2018.

[13] M. Al-Jabi and H. Sammaneh, "Toward mobile ar-based interactive smart parking system," 2018 IEEE 20th International Conference on High Performance Computing and Communications; IEEE 16th International Conference on Smart City; IEEE 4th International Conference on Data Science and Systems (HPCC/SmartCity/DSS), vol. PP. 1243-1247, 2018.

[14] D. Kanteti, D. V. S. Srikar, and T. K. Ramesh, "Intelligent smart parking algorithm," 2017 International Conference On Smart Technologies For Smart Nation (SmartTechCon), vol. PP. 1018-1022, 2017.

[15] T. Lin, H. Rivano, and F. Le Mouël, "A survey of smart parking solutions," IEEE Transactions on Intelligent Transportation Systems, vol. Vol. 18, No. 12, PP. 3229-3253, 2017.

[16] K. S. Awaisi, A. Abbas, M. Zareei, H. A. Khattak, M. U. Shahid Khan, M. Ali, I. Ud Din, and S. Shah, “Towards a fog enabled efficient car parking architecture," IEEE Access, vol. Vol. 7, PP. 159100-159111, 2019.

[17] C. Tang, X. Wei, C. Zhu, W. Chen, and J. J. P. C. Rodrigues, "Towards smart parking based on fog computing," IEEE Access, vol. Vol. 6, PP, 70172-70185, 2018.

[18] C. Shi, J. Liu, and C. Miao, "Study on parking spaces analyzing and guiding system based on video," IEEE, 23rd International Conference on Automation and Computing (ICAC), vol. pp. 1 - 5, sept., 2017.

[19] M. Turk and A. Pentland, "Eigenfaces for recognition," Journal of Cognitive Neuroscience, vol. vol.3, no.1, pp. 71-86, 1991.

[20] R. S. Sabeenian, "Hand written text to digital text conversion using radon transform and back propagation network (rtbpn)," International Journal of Computers Information Technology and Engineering (IJCITAE), vol. Vol. 101, pp. 498 - 500, 2010.

[21] M. M. MohieEl-din, N. I. Ghali, A. G. Ahmed, and H. A. El-Shenbary, "A study on the impact of wavelet decomposition on face recognition methods," International Journal of Computer Applications (IJCA), vol. vol. 87, no. 3, pp. 14 - 21, Feb. 2014.

[22] M. M. MohieEl-din, M. Y. El-Nahas, and H. A. El-Shenbary, "Hybrid framework for robust multimodal face recognition," International Journal of Computer Science Issues (IJCSI), vol. vol. 10, Issue 2, no. 2, pp. 471 - 476, Mar. 2013.

[23] M. S. Farag, M. M. M. E. Din, and H. A. E. Shenbary, "Parking entrance control using license plate detection and recognition," Indonesian Journal of Electrical Engineering and Computer Science(IJEECS, vol. vol.15, No. 1, pp. $476-483,2019$.

[24] P. Almeida, L. S. Oliveira, J. E. Silva, J. A. Britto, and A. Koerich, "Pklot - a robust dataset for parking lot classification," Expert Systems with Applications, vol. vol.42,No.11, pp. 4937-4949, 2015.

[25] A. Giuseppe, C. Fabio, F. Fabrizio, G. Claudio, M. Carlo, and V. Claudio, "Deep learning for decentralized parking lot occupancy detection,” Expert Systems with Applications, vol. 72, pp. 327-334, 2017.

[26] R. Girshick, J. Donahue, T. Darrell, and J. Malik, "Rich feature hierarchies for accurate object detection and semantic segmentation," In Proceedings of the ieee conference on computer vision and pattern recognition, vol. pp. 580-587, 2014.

[27] A. Krizhevsky, S. Ilya, and H. E. Geoffrey, "Imagenet classification with deep convolutional neural networks," Advances in neural information processing systems, vol. pp. 1097-1105, 2012. 